\title{
Editorial of special section on multimedia applications and ubiquitous computing
}

\author{
Tai-hoon Kim • Sabah Mohammed
}

Published online: 9 May 2013

(C) Springer Science+Business Media New York 2013

On behalf of the Science and Engineering Research Support soCiety-SERSC, it is an honor for us to introduce you to this special section, which includes a selection of the best papers presented at the 3rd International Conference on Multimedia, Computer Graphics and Broadcasting (MulGraB 2011) and the 3rd International Conference on Ubiquitous Computing and Multimedia Applications (UCMA 2012), as well as other articles that cover topics of particular interest to researchers in the fields of Multimedia Applications and Ubiquitous Computing.

This issue contains 10 articles coming from various countries, among which we mention Japan, Australia, South Korea, Canada and China. Achieving such a high quality of papers would have been impossible without the huge work that was undertaken by the Editorial Board members and External Reviewers. We take this opportunity to thank them for their great support and cooperation.

In "Discover and Visualize Association Rules from Sensor Observations on the Web," authors focused on using data mining approaches to achieve knowledge discovery. Authors selected association rule analysis as the approach to discover relationships and patterns among different sensor observations. Compared with traditional conceptual models, the proposed approach by authors had lower requirements on data quality and domain expertise.

In "Recommendation Algorithm of the App Store by using Semantic Relations between Apps," authors proposed a personalized recommendation system for mobile application software (app) to mobile user using semantic relations of apps consumed

T.-h. $\operatorname{Kim}(\bowtie)$

University of Tasmania, Sandy Bay, Australia

e-mail: taihoonn@daum.net

S. Mohammed

Lakehead University, Thunder Bay, Canada 
by users. To do that, authors defined semantic relations between apps consumed by a specific member and his/her social members using ontology. Based on the relations, authors identified the most similar social members from the reasoning process.

Authors proposed a convergence indicator obtained from patent citation analysis as a method of analyzing the basis of more universal interpretation in "Convergence Indicator: The Case of Cloud Computing." The method used in this paper involves a microscopic analysis based on the individual citation relationship of patents, rather than a macroscopic technological convergence analysis such as a statistical approach. The use of such a method can minimize the operational definition in the study of convergence, and allow relative comparison and analysis of the convergence level of the patents to be analyzed.

In "A Mechanism for Achieving a Bound on Execution Performance of Process Group to Limit CPU Abuse," authors proposed an improved mechanism for achieving a bound on the execution performance of process groups in order to limit unnecessary processor use. Authors improved the previously proposed mechanism used controlling the upper bound for a process. Also, authors introduced directory execution as an execution resource with an upper bound.

In the paper "Towards Designing a Secure Biosurveillance Cloud," authors utilized cloud computing as a low-cost computational platform to address critical challenges and limitations in today's surveillance systems and to introduce design concepts to facilitate the security in such systems. Authors identified several security components to build a trusted environment for cloud-based biosurveillance system by integrating the legacy health enterprises private clouds with the public clouds based on the distributed OSGi framework along with a distributed authentication service.

In "Development of a Virtualized Supercomputing Environment for Genomic Analysis," authors presented the design and implementation of a virtualized supercomputing environment that provides functions for virtual cluster management and job scheduling in a virtual cluster pool. Authors also presented a virtualized system architecture that takes data locality into account for the data intensiveness of NGS analysis. The virtualized environment enhanced the resource utilization and scalability for NGS applications. Also, authors evaluated the performance and scalability of the virtualized environment using a representative NGS application workload.

Authors proposed a new transcoding proxy mechanism, the Fuzzy Similaritybased Transcoding Proxy (FSTP), to solve the problems of existing schemes in "Streaming Media Service based on Fuzzy Similarity in Wireless Mobile Networks." The proposed FSTP mechanism calculates the fuzzy similarity degree for partitioned segment versions of streaming media objects to construct the Fuzzy Similarity-based Transcoding Graph (FSTG).

In "A Framework for Online Gait Recognition based on Multilinear Tensor Analysis," authors proposed an online framework for gait recognition based on the multilinear tensor analysis. First of all, for discriminative analysis of cyclic characteristic which gait sequence has, the gait cycle is divided into four segments according to the peaks of the stride length variation and, then, all of silhouettes within each interval are integrated into one frame to form the accumulated silhouettes. All of the silhouettes in the cycle are also integrated to form another accumulated silhouette which reflects the whole characteristic of the gait sequence. 
In the shape-based gait recognition, the dependency on the direction of walking is a very serious problem. As one of possible solutions for these dependencies, it is a noticeable approach to synthesize gait sequences to the canonical-viewed ones in order to extract steady features from the gait sequences. To reduce the dependency on the direction of walking, in the paper "Gait Recognition Using Description of Shape Synthesized by Planar Homography," authors proposed a view-synthesis method based on the planar homography which not only transforms the oblique-directional gait to the canonical-viewed one but also normalizes the size of silhouette with simple operation regardless the position.

To decrease isolated nodes (a device may become an isolated node due to constraints of configuration parameters in the ZigBee network), in "An Innovative Scheme for Increasing Connectivity and Life of ZigBee Networks," authors proposed an approach called Enhanced Connectivity Scheme for the ZigBee network. Authors showed this scheme can efficiently reconstruct parts of the network to let more devices join and hence increase the join ratio.

We would like to thank the authors and reviewers of all of the manuscripts that were submitted. Last but not least, we would like to thank Professor Hamid R. Arabnia, Editor-in-Chief, for his stimulating encouragements and the staff of SpringerVerlag for prompt assistance and excellent organization skills in helping to meet the deadlines and provide our association with this invaluable opportunity to show and disseminate the research activities.

We hope that the readers of Journal of Supercomputing will find this special section interesting and useful.

April, 2013

Tai-hoon Kim

Sabah Mohammed 